Case Report

\title{
Native Valve Streptococcus bovis Endocarditis and Refractory Transfusion Dependent Iron Deficiency Anaemia Associated with Concomitant Carcinoma of the Colon: A Case Report and Review of the Literature
}

\author{
Abdul Azeez Ahamed Riyaaz, ${ }^{1}$ Randula Samarasinghe, ${ }^{1}$ Kolitha Sellahewa, \\ Sabaratnam Sivakumaran, ${ }^{1}$ and Manjula Sri Tampoe ${ }^{2}$ \\ ${ }^{1}$ Department of Internal Medicine, Dr Neville Fernando Teaching Hospital, Millennium Drive, Chandrika Kumaratunga Mawatha, \\ 10115 Malabe, Sri Lanka \\ ${ }^{2}$ South Asian Institute of Technology and Medicine, Millennium Drive, Chandrika Kumaratunga Mawatha, 10115 Malabe, Sri Lanka
}

Correspondence should be addressed to Abdul Azeez Ahamed Riyaaz; ahamed.riyaaz@gmail.com

Received 25 September 2015; Accepted 16 December 2015

Academic Editor: Pau Montesinos Fernández

Copyright (C) 2016 Abdul Azeez Ahamed Riyaaz et al. This is an open access article distributed under the Creative Commons Attribution License, which permits unrestricted use, distribution, and reproduction in any medium, provided the original work is properly cited.

\begin{abstract}
Streptococcus bovis is found as a commensal organism in human gut and may become opportunistically pathogenic. Infective endocarditis is one of the commonest modes of presentation of this infection. The association between Streptococcus bovis endocarditis and colorectal cancer is well recognized. We report a case of Streptococcus bovis endocarditis along with a refractory iron deficiency anaemia associated with concomitant carcinoma of ascending colon in a 63-year-old male. Cooccurrence of these two conditions may cause a challenge in the management. Considering the strong association of colon cancer with Streptococcus bovis endocarditis, a detailed screening colonoscopy is mandatory following the diagnosis of the latter.
\end{abstract}

\section{Introduction}

The gastrointestinal tract in 2.5 to $15 \%$ of human population is colonized by Streptococcus bovis. Bacteremia and endocarditis are the commonest presentation of infection by this organism when they become pathogenic. Streptococcus bovis accounts for around $13 \%$ of all infective endocarditis cases and usually causes severe valve damage. The association of Streptococcus bovis and gastrointestinal pathologies, colon cancer in particular, has been well established. The aim of this report is to highlight the clinical, diagnostic, and management aspects of a patient who presented with Streptococcus bovis endocarditis and a refractory iron deficiency anaemia followed by the detection of a concomitant colon cancer.

\section{Case Presentation}

A 63-year-old man from western part of Sri Lanka with a long-standing history of type 2 diabetes mellitus and hypertension admitted to our medical unit with an acute febrile illness with duration of 2 days. He complained of generalized malaise, anorexia, and weight loss over the last 4 months. Upon systemic inquiry, he did not have any symptoms related to respiratory, gastrointestinal, and genitourinary systems.

He had been under investigation for symptomatic iron deficiency anaemia with no identified cause over the last 4 months which required 2 hospital admissions and 8 blood transfusions. An upper gastroscopy was normal and he was advised to undergo a colonoscopy during his last hospital admission 2 months ago but he refused the procedure. He was discharged and was on oral iron supplements until his current hospital admission.

On examination, he was looking ill and febrile (temperature: $38.3^{\circ} \mathrm{C}$ ). There was severe pallor of his conjunctivae without icterus, lymphadenopathy, skin rashes, or peripheral stigmata of infective endocarditis. Cardiovascular 


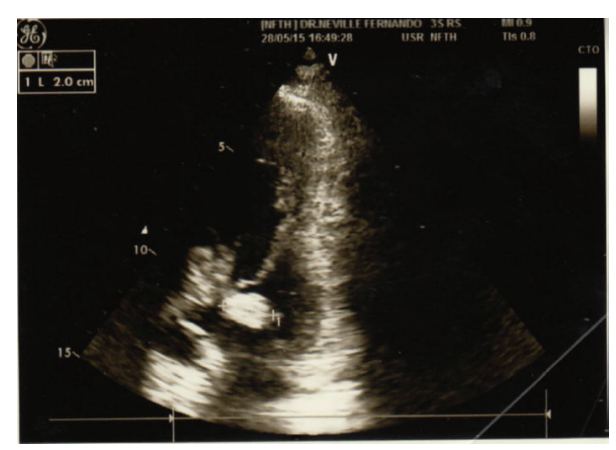

Figure 1: 2D echocardiogram showing large sessile vegetation attached to anterior mitral valve leaflet.

examination revealed a pulse rate of 96 beats per minute, blood pressure of $140 / 90 \mathrm{~mm} \mathrm{Hg}$, a normal JVP, slightly deviated apex beat, and normal 1st and 2nd heart sounds without any additional heart sounds or murmurs. Respiratory system examination was unremarkable and his respiratory rate was 18 breaths per minute. There was an asymmetrical swelling of the right side of his abdomen, deep palpation of which revealed a $10 \mathrm{~cm}$ sized nontender, smooth, and rounded mass in the right upper quadrant.

Initial investigations showed haemoglobin of $5.9 \mathrm{~g} / \mathrm{dL}$, white cell count of $9.22 \mathrm{k} / \mathrm{mm}^{3}$ with $63 \%$ neutrophils, platelets of $360 \mathrm{k} / \mathrm{mm}^{3}$, C-reactive protein of $277 \mathrm{mg} / \mathrm{dL}$, normal renal and liver function tests, and a normal chest X-ray. A blood culture taken on admission grew group D Streptococcus species after 24 hours of incubation which was identified as Streptococcus bovis subsequently. He was started on intravenous Ceftriaxone $2 \mathrm{~g}$ daily. On the 3 rd day of his hospital admission, he developed a sudden onset left sided hemiparesis and dysarthria which resolved completely within few hours. A noncontrast CT brain did not show any infarction or haemorrhage. Transient ischaemic attack due to possible septic emboli was suspected and 2D echocardiogram was requested which showed a large $(18 \mathrm{~mm})$ sessile vegetation attached to the anterior mitral valve leaflet (Figure 1) associated with grade 2 mitral regurgitation and normal left ventricular systolic function. Following the diagnosis of Streptococcus bovis endocarditis antibiotic was changed to intravenous benzyl penicillin 4 mu every 6 hours and gentamycin $1 \mathrm{mg} / \mathrm{kg}$ every 8 hours. An Abdominal CT scan was done in view of right sided abdominal mass which showed the mass was arising from the ascending colon near hepatic flexure with no metastases to the liver. The patient underwent a colonoscopy which confirmed the growth at the level of hepatic flexure (Figure 2), biopsy of which revealed an adenocarcinoma. His fever settled after the 5th day of antibiotic therapy with serial reduction in inflammatory markers indicating resolution of endocarditis. But he developed frank bleeding per rectum and progressive increase in blood transfusion requirements during the 3rd week of his illness. After a multidisciplinary team discussion involving gastroenterology surgeon, oncologist, and cardiothoracic surgeon it was decided to proceed with an urgent surgery to remove the tumour due to active bleeding although a cardiothoracic surgery too was indicated at that

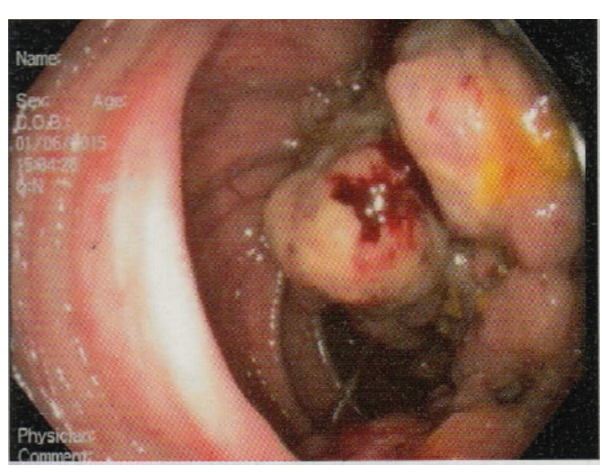

FIGURE 2: Colonoscopy image of the ascending colon at the level of hepatic flexure showing the tumour.

TABLE 1: Nomenclature of the principal human species of Streptococcus bovis.

\begin{tabular}{ll}
\hline Old nomenclature & Recent nomenclature \\
\hline Streptococcus bovis biotype I & S. gallolyticus subsp. gallolyticus \\
Streptococcus bovis biotype II/1 & S. infantarius subsp. infantarius \\
S. infantarius subsp. coli
\end{tabular}

time due to persistent large vegetation and thromboembolic risk. He underwent an extended right hemicolectomy at the end of 5th week of his illness. It was a large tumour arising from the caecum and extending up to hepatic flexure with a staging of $\mathrm{T}_{3} \mathrm{~N}_{0} \mathrm{M}_{0}$ at surgery, biopsy of which revealed a moderately differentiated adenocarcinoma. He had an uneventful recovery and was closely observed and followed up for thromboembolic complication from the vegetation. Follow-up echocardiogram revealed a healed and slightly calcified vegetation with moderate mitral regurgitation without significant left ventricular dysfunction. He is currently awaiting a mitral valve replacement surgery.

\section{Discussion}

Streptococcus bovis is found as a commensal organism in 2.5$15 \%$ of normal population [1]. It is nonenterococcal Lancefield group D Streptococcus for which a new nomenclature exists (Table 1) [2]. S. bovis is the second commonest streptococcal species to cause infective endocarditis and is responsible for around $13 \%$ of all infective endocarditis cases [3]. The portal of entry for this organism is usually the gastrointestinal tract and less commonly the biliary and urinary tracts. Although previous literature reported high prevalence of simultaneous multivalvular involvement in $S$. bovis endocarditis, a very recent single center study shows the prevalence is only around $28 \%$. Our patient had a single valve involvement with a large vegetation and mild valve damage [4]. Higher incidence of neurological complications occurs with $S$. bovis endocarditis and the incidence of stroke is reported to be around $6 \%$ [5]. Our patient also developed a transient ischaemic attack possibly due to a septic embolism from the vegetation. 
The association between streptococcal endocarditis including S. bovis and colon cancer was first described in early 1950s by McCoy and Mason [6]. After 2 decades, Hoppes and Lerner reported 14 cases of $S$. bovis endocarditis in which 9 (64\%) cases were associated with concomitant gastrointestinal disease [7]. This association was further strengthened by the findings that the faecal carriage rate for this $S$. bovis was 5 times higher in patients with colonic malignancy compared with normal population and around half of patients with colon cancer develop silent infection with S. bovis [8]. In recent systematic review and metaanalysis authors report it is Streptococcus bovis biotype I (Streptococcus gallolyticus) which has the unambiguous association with gastrointestinal disease compared to other subspecies [9]. The reported incidence of colon cancer in patients with S. bovis endocarditis varies between 18 and $62 \%$ [10]. Other gastrointestinal pathologies reported with this association include benign colonic lesions, gastric cancer, peptic ulcer disease, inflammatory bowel disease, and liver cirrhosis [11].

It has been controversial whether the association between $S$. bovis bacteremia and endocarditis with colon cancer is a consequence of gastroenterological lesion or it could be related to its aetiopathogenesis. A recent review of research in this field shows this association is aetiological in nature. Cytokines such as IL-1, IFN-gamma, and IL-8, produced by $S$. bovis infection, are most probably responsible for a slow progressing carcinogenesis of colorectal mucosal tissues which appears to occur through a transformation process from normal tissues to premalignant lesions and finally to malignant tissues [12]. Proinflammatory potentials of $S$. bovis including the leucocytic recruitment, the tumor tissueselective adhesion potential, the selective colonization in tumor cells, the suitable microenvironment of tumor tissues for S. bovis proliferation, and the local disruption of tumor tissues and capillaries which allow the entry of $S$. bovis into blood circulation seem to contribute to bacteremia [12].

The management of $S$. bovis endocarditis is straightforward as in other streptococcal endocarditis once the diagnosis is made. Emergency or urgent valve replacement surgery may be indicated in many cases since the incidence of haemodynamically unstable valve damage and neurological complications are common with $S$. bovis endocarditis. Management dilemma may arise as in our patient if there is concomitant colon cancer which warrants urgent surgery particularly when both bowel and cardiothoracic surgery are indicated in relation to preference and timing of surgery. We could not find any publications or studies addressing these issues in our literature search. Thus it is extremely useful to involve multidisciplinary team including gastroenterology surgeon, oncologist, and cardiothoracic surgeon in the decision-making process.

\section{Conclusions}

Considering the strong association between S. bovis septicaemia and/or endocarditis and colonic carcinoma it cannot be overemphasized that a detailed colonoscopy is mandatory in the assessment of these patients. Moreover, unexplained iron deficiency anaemia as in our patient is an additional indication for colonoscopy as it is a well-recognized presentation of occult right sided colonic malignancy. Further research is needed to address the management issues when S. bovis endocarditis and colon malignancy coexist.

\section{Abbreviations \\ JVP: Jugular venous pressure \\ CT: Computerized tomography \\ S. bovis: Streptococcus bovis \\ IL: Interleukin \\ IFN: Interferon.}

\section{Consent}

Written informed consent was obtained from the patient for the publication of this case report and accompanying images.

\section{Conflict of Interests}

The authors declare that they have no conflict of interests.

\section{Authors' Contribution}

Abdul Azeez Ahamed Riyaaz, Randula Samarasinghe, Kolitha Sellahewa, and Sabaratnam Sivakumaran were involved in the diagnosis and management of the patient. Abdul Azeez Ahamed Riyaaz and Manjula Sri Tampoe were involved in writing the paper. Kolitha Sellahewa reviewed and approved the final version of the paper.

\section{Acknowledgments}

The authors would like to thank the cardiology, gastroenterology, microbiology, and radiology departments of Dr Neville Fernando Teaching Hospital and the National Hospital of Sri Lanka for their extended support in the diagnosis and management of this patient.

\section{References}

[1] L. V. Hooper and J. I. Gordon, "Commensal host-bacterial relationships in the gut," Science, vol. 292, no. 5519, pp. 1115-1118, 2001.

[2] L. Schlegel, F. Grimont, E. Ageron, P. A. D. Grimont, and A. Bouvet, "Reappraisal of the taxonomy of the Streptococcus bovis/Streptococcus equinus complex and related species: description of Streptococcus gallolyticus subsp. gallolyticus subsp. nov., S. gallolyticus subsp. macedonicus subsp. nov. and S. gallolyticus subsp. pasteurianus subsp. nov," International Journal of Systematic and Evolutionary Microbiology, vol. 53, no. 3, pp. 631-645, 2003.

[3] I. Kupferwasser, H. Darius, A. M. Müller et al., "Clinical and morphological characteristics in Streptococcus bovis endocarditis: a comparison with other causative microorganisms in 177 cases," Heart, vol. 80, no. 3, pp. 276-280, 1998. 
[4] J. Corredoira, M. J. García-País, A. Coira et al., "Differences between endocarditis caused by Streptococcus bovis and Enterococcus spp. and their association with colorectal cancer," European Journal of Clinical Microbiology \& Infectious Diseases, vol. 34, no. 8, pp. 1657-1665, 2015.

[5] M. Ballet, G. Gevigney, J. P. Garé, F. Delahaye, J. Etienne, and J. P. Delahaye, "Infective endocarditis due to Streptococcus bovis. A report of 53 cases," European Heart Journal, vol. 16, no. 12, pp. 1975-1980, 1995.

[6] W. C. McCoy and J. M. Mason, "Enterococcal endocarditis associated with carcinoma of the sigmoid; report of a case," Journal of the Medical Association of the State of Alabama, vol. 21, no. 6, pp. 162-166, 1951.

[7] W. L. Hoppes and P. I. Lerner, "Nonenterococcal groupD streptococcal endocarditis caused by Streptococcus bovis," Annals of Internal Medicine, vol. 81, no. 5, pp. 588-593, 1974.

[8] R. S. Klein, R. A. Recco, M. T. Catalano, S. C. Edberg, J. I. Casey, and N. H. Steigbigel, "Association of Streptococcus boviswith carcinoma of the colon," The New England Journal of Medicine, vol. 297, no. 15, pp. 800-802, 1977.

[9] A. Boleij, M. M. H. J. van Gelder, D. W. Swinkels, and H. Tjalsma, "Clinical importance of Streptococcus gallolyticus infection among colorectal cancer patients: systematic review and meta-analysis," Clinical Infectious Diseases, vol. 53, no. 9, pp. 870-878, 2011.

[10] C. Leport, A. Bure, J. Leport, and J. L. Vilde, "Incidence of colonic lesions in Streptococcus bovis and enterococcal endocarditis," The Lancet, vol. 329, no. 8535, p. 748, 1987.

[11] J. G. Reynolds, E. Silva, and W. M. McCormack, "Association of Streptococcus bovis bacteremia with bowel disease," Journal of Clinical Microbiology, vol. 17, no. 4, pp. 696-697, 1983.

[12] A. S. Abdulamir, R. R. Hafidh, and F. A. Bakar, "The association of Streptococcus bovis/gallolyticus with colorectal tumors: the nature and the underlying mechanisms of its etiological role," Journal of Experimental and Clinical Cancer Research, vol. 30, article 11, 2011. 


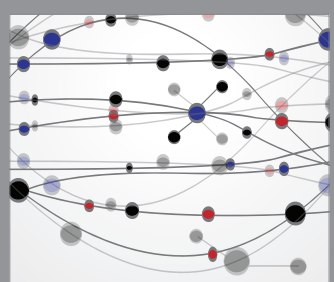

The Scientific World Journal
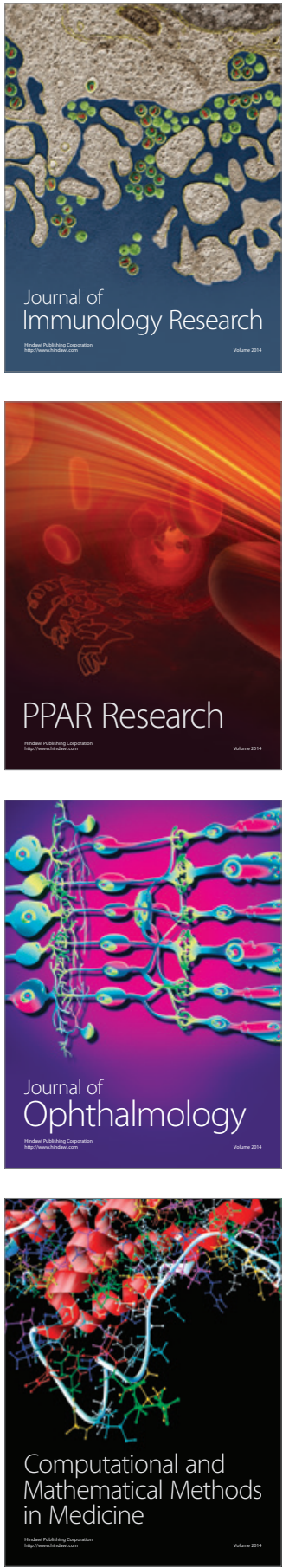

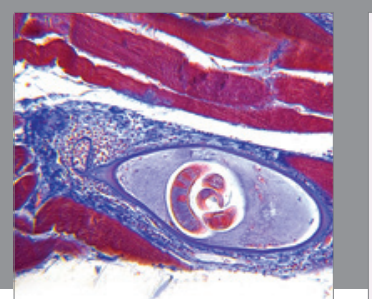

Gastroenterology Research and Practice

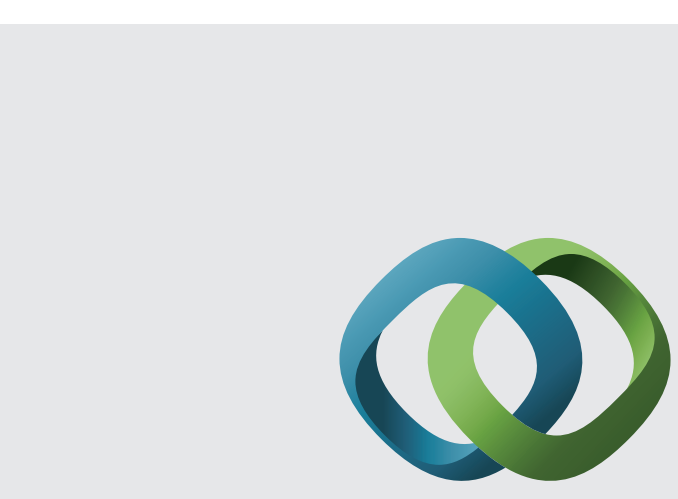

\section{Hindawi}

Submit your manuscripts at

http://www.hindawi.com
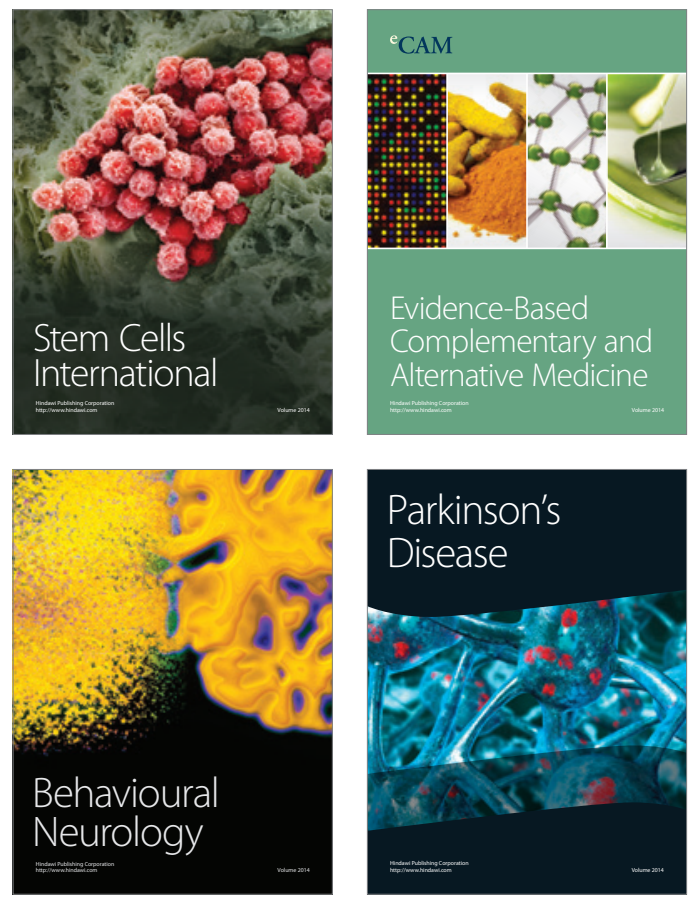
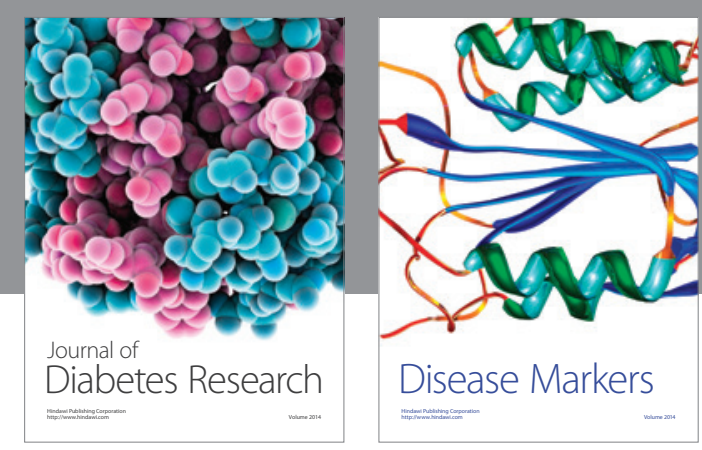

Disease Markers
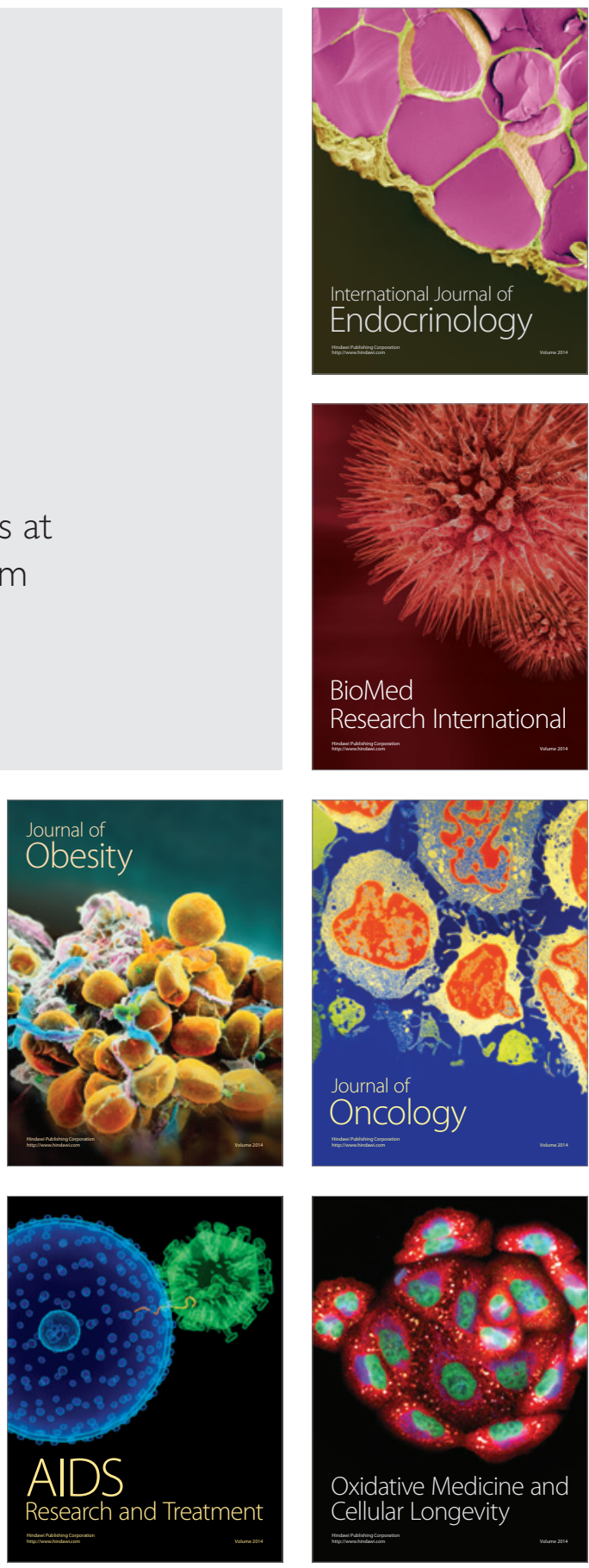